\title{
The Effect of Endometrial Scratching by Pipelle on the Pregnancy Rate in Couples with Unexplained Infertility
}

\author{
Belal S ${ }^{2 *}$, Nagy OAEFA ${ }^{1}$, Elwanis $\mathrm{MA}^{2}$ and \\ Mahmod $\mathbf{H}^{2}$ \\ ${ }^{1}$ Reproductive Health Research Department, National \\ Research Center, Egypt \\ ${ }^{2}$ Department of Obstetrics and Gynecology, El Sahel \\ Teaching Hospital, Egypt \\ *Corresponding author: Shimaa Belal, Department \\ of Obstetrics and Gynecology, Elsahel Teaching Hospital, \\ Egypt
}

Received: November 15, 2017; Accepted: December 08, 2017; Published: December 15, 2017

\begin{abstract}
Background: Unexplained Infertility (UI) is a term used to describe infertile couples in whom standard investigations, including semen analysis, tests of ovulation and tubal patency, have failed to detect any gross abnormality. Endometrial scratching could have a favorable endometrial healing effect on the implantation process by releasing of biochemical mediators such as endometrial pro inflammatory cytokines (interleukin-6, leukemia inhibitory factor and tumor necrosis factor) that characterize early implantation. These cytokines can be secreted by the endometrial cells and cells of the immune system. Endometrial scratching has been suggested to boost embryo implantation following recurrent implantation failure after IVF.
\end{abstract}

Objectives: The aim of this study is to compare the endometrial scratching by pipelle to the non pipelle endometrial scratching as regards biochemical pregnancy rate in women with unexplained infertility.

Methodology: 150 patients aged between 20-35y will be recruited from women having unexplained infertility for more than a year attending outpatients clinics in El Sahel Teaching Hospital. Random sequence will be generated using MS Excel by second supervisor. Patients will be distributed according to randomization card by preparing 3 groups by closed enveloped card $A, B$, C. (75 patients in each group): Group A will be the control group, group B will include participants submitted to pipelle intervention and group $C$ will include the participants submitted to uterine sound intervention group. Combined oral contraceptive pills that contain norgestimate $0.25 \mathrm{mg}$, ethinyl oestradiol $0.35 \mathrm{mg}$ (cilest ${ }^{\circ}$, janssen, Cairo, Egypt) is taken by each participant at the first day of the same cycle of intervention for twenty once days and were followed for six months for incidence of pregnancy.

Results: The rate of biochemical pregnancy was significantly higher in women subjected to endometrial injury by Pipelle compared with non endometrial injury by sound and control group. The rate of biochemical pregnancy in the interventional group (Pipelle \& sound) was significantly higher than control group.

Conclusion: Local mechanical injury to the endometrium can enhance uterine receptivity probably by provoking the production of molecules and inflammatory mediators that facilitate the implantation of the embryo and thus improvement of ongoing pregnancy. This procedure is simple, easy, low cost and effective in unexplained infertility.

Recommendations: According to the results of this study, and the beneficial effect of endometrial scratching on implantation rate and pregnancy rate in women with unexplained infertility, endometrial scratching can be done in spontaneous cycle of these women before invasive interventions as IUI, IVF and laparoscopy this may help to reduce psychological tensions and high expenses imposed through such interventions.

\section{Introduction}

Endometrial injury is a simple, minimally invasive, low cost procedure that may boost biochemical and clinical pregnancy rates, the endometrial is gently 'scratched' using the hysteroscopy or a thin catheter (a fine, flexible, sterile, plastic tube) which is passed through the cervix [1].

In the studies of [2], endometrial injury was performed approximately 2 to 3 weeks prior to embryo transfer, thus representing the shortest period associated with a positive effect of LEI on endometrial receptivity. Additionally [3] showed that endometrial injury on the day of oocyte retrieval was detrimental to the IVF success rate. This suggests that endometrial injury very close to the time of embryo transfer could have a negative impact, since it would not allow sufficient time for endometrial regeneration and proliferation to achieve the desired effect. 
The procedure was conducted at the luteal phase on days 21-26 of the spontaneous menstrual cycle, endometrial samples were obtained using a biopsy catheter (pipelle), after introduction of the pipelle into the uterine cavity, it was rotated 360 degrees and moved up and down four times after withdrawing the piston [4,5]. There is a beneficial effect of endometrial scratching in terms of biochemical pregnancy rate and clinical pregnancy rate in women with unexplained infertility, the biochemical and clinical pregnancy rate were higher in women in the interventional group (Pipelle group) than women in the control group (Sound group) [5]. A randomized controlled trial concluded that total of 217 women with unexplained infertility were randomly divided into two study groups. After super ovulation by clomiphenecitrate and gonadotropins and when the dominant follicles reached $18-20 \mathrm{~mm}$, patients were randomly assigned to undergo endometrial local injury at posterior uterine wall by pipelle, then all the patients were instructed to follow a regularly timed intercourse, the pregnancy rate was significantly higher in the endometrial injury group compared to the control group [6].

In [7] induced endometrial injury in the luteal phase on days 21 and 26 among 60 women with implantation failure in IVF cycle was done, they compared the results with 57 others who did not undergo endometrial injury, the implantation and pregnancy rates in the injury group were higher than in the control group.

\section{Subjects and Methods}

The study was an interventional prospective randomized controlled clinical trial included 150 women age ranging between 20-35y with unexplained infertility recruited from outpatients' clinics in El Sahel Teaching Hospital during the period from July 2015 to June 2016 to compare the effect of endometrial scratching by using Pipelle with non endometrial scratching for women with unexplained infertility after obtaining approval of El Sahel Teaching Hospital and a written informed consent from the patient participating in the research.

Inclusion criteria for cases included (Women diagnosed to have unexplained infertility as semen analysis, assessment of ovulation and tubal patency fail to detect any gross abnormality in a woman who has not conceived for one year of unprotected sexual intercourse, regular menstruation with the length of the cycle between 21-35 days, ovulation confirmed by appropriately timed mid-luteal progesterone (3-10ng/mL). day $3 \mathrm{FSH}$ level less than $10 \mathrm{mIU} / \mathrm{ml}$, bilateral tubal patency (demonstrated by laparoscopy or hystrosalpingography). Exclusion Criteria for cases included: Patients aged less than $20 \mathrm{y}$ or more than 35y, infertile semen analysis, day 3 FSH more than 10mIU/ $\mathrm{ml}$, medical disorders as (Polycystic Ovary (PCO), hyperthyroidism, hypothyroidism, diabetes mellitus, hypertension, liver and renal diseases), pelvic pathology as PID or uterine fibroids. previous history or planning for IVF, and not receiving drugs inducing ovulation in last six months, history of smoking to be identified.

At first visit, general data will be obtained for criteria of eligibility. Then, historical data will be collected including menstrual cycle and related history, medical disorders as Polycystic Ovary (PCO), hyperthyroidism, hypothyroidism, diabetes mellitus, hypertension, liver and renal diseases, any recent use of hormonal medications that could affect the patients cycle.
Combined oral contraceptive pills that contain norgestimate $0.25 \mathrm{mg}$, ethinyl oestradiol $0.35 \mathrm{mg}$ (cilest', janssen, Cairo, Egypt) is taken by each participant at the first day of the same cycle of intervention for twenty once days and were followed for six months for incidence of pregnancy. Random sequence will be generated using MS Excel by second supervisor. Patients will be distributed according to randomization card by preparing 3 groups by closed enveloped card A, B, C. (50 patients in each group): Group A will be the control group, group B will include participants submitted to pipelle intervention and group $\mathrm{C}$ will include the participants submitted to uterine sound intervention group. In control group (group A) no intervention will be done. Endometrial scratching will be performed for women in the Pipelle group (group B) on outpatient clinic. After introduction of the pipelle (Genetics medical Products, HamonalAchel, Belgium) into the uterine cavity at the luteal phase on days 21-26 of spontaneous menstrual cycles, it will be rotated 360 degree and moved up and down four times after withdrawing the piston. For women in the third group (group C). The uterine sound will be introduced into the uterine cavity without endometrial scratching at the luteal phase on days 21-26 of spontaneous menstrual cycles. Couples will be advised to practice sexual intercourse according to their convenience for the next 6 months and couples in the three groups will be asked to phone a contact person whenever there was a missed period during the follow up. In the presence of missed period follow up of each participant by B-HCG and ultrasound to confirm intrauterine pregnancy. Table 1 shows that there were statistical significant differences between women in Pipelle group, sound group and control group as regard incidence of pregnancy but there no were statistical significant differences between the three groups as regard mean age, BMI, duration of infertility, FSH, LH, E2. Figure 3 shows the rate of biochemical pregnancy that was significantly higher in women in Pipelle group than (sound \& control) group ( $30 \%$ vs. $10 \% \& 8 \%$ respectively), $(\mathrm{p}<0.05=0.001)$. Figure 4 shows the rate of biochemical pregnancy that was higher in women in the interventional group (Pipelle \& sound) than control group $\{20 \%$ vs $8 \%\}$. Interventional group showed significantly higher rate $(\mathrm{p}<0.05$ $=0.045)$.

\section{Data Analysis}

Statistical analysis will be done on a personal computer using IBM $^{\odot}$ SPSS $^{\oplus}$ Statistics version 21 (IBM ${ }^{\odot}$ Corp., Armonk, NY, USA). Data will be collected, tabulated then analyzed using appropriate statistical tests. The D'Agostino-Pearson test will be used to test the normality of numerical data distribution one-way analysis of variance. (ANOVA) (For multiple - group comparison). Numerical data will be presented as mean, standard deviation and median. Categorical data will be presented as number and percentage or as ratio. The chi square test or (Fisher's exact test, when appropriate) will be used to compare categorical data.

\section{$\mathrm{P}<0.05$ will be considered statistically significant.}

\section{Results}

Results of the study are summarized in the (Table 1) and (Figure $3,4)$. Regarding the rate of biochemical pregnancy, was significantly higher in women subjected to endometrial injury by Pipelle (15/50) compared with non endometrial injury by sound (5/50) and control 


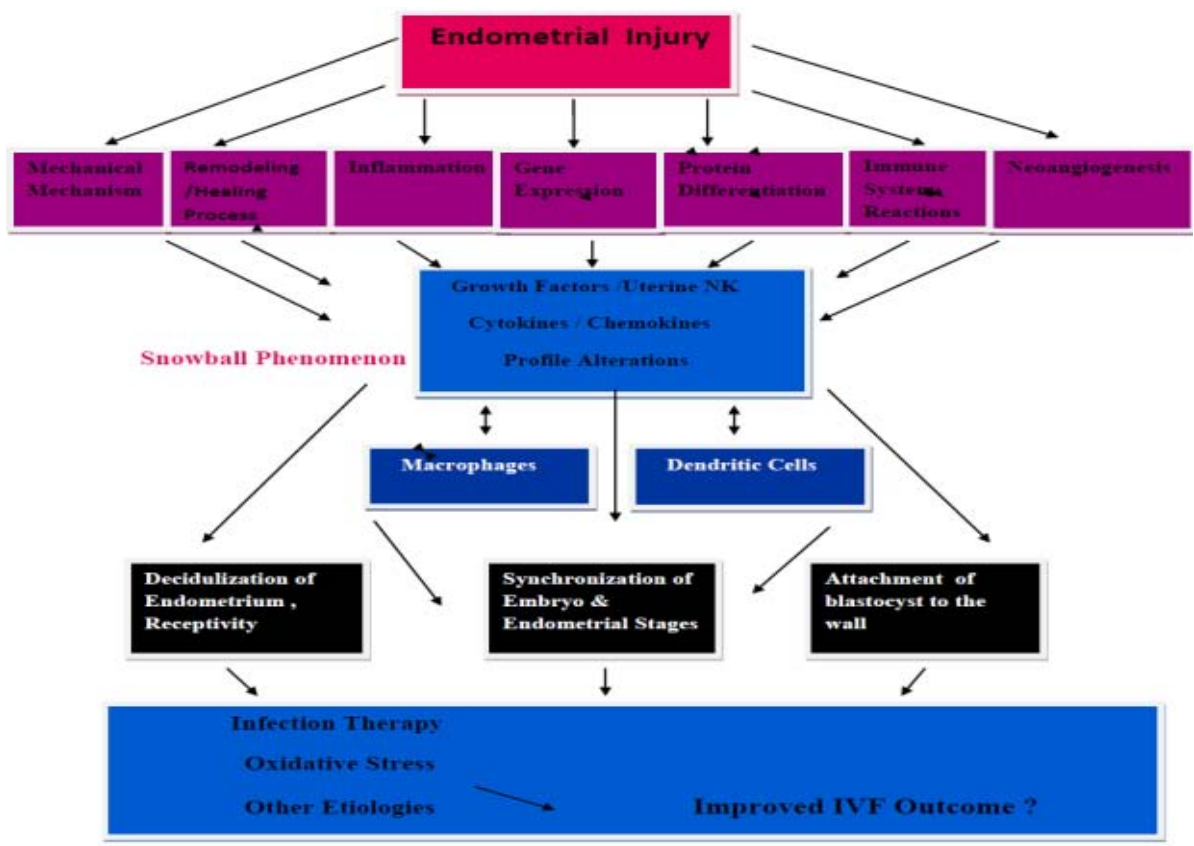

Figure 1: Schematic representation of the mechanisms involved in endometrial injury [19].

Table 1: Patients' characteristics in the control, uterine sound and pipelle groups.

\begin{tabular}{|c|c|c|c|c|}
\hline Variable & Control group $(n=50)$ & Uterine sound group $(n=50)$ & Pipelle group $(n=50)$ & p-value \\
\hline Age (yr) & $28.9 \pm 3.9$ & $27.4 \pm 4.7$ & $26.7 \pm 4.3$ & $0.135 \pi$ \\
\hline BMI $\left(\mathrm{kg} / \mathrm{m}^{2}\right)$ & $27.3 \pm 4.1$ & $27.5 \pm 3.9$ & $27.6 \pm 3.8$ & $0.961 \pi$ \\
\hline Duration of infertility (yr) & $2.9 \pm 2.6$ & $2.6 \pm 1.7$ & $2.8 \pm 2.1$ & $0.225 \pi$ \\
\hline $\mathrm{FSH}(\mathrm{mlU} / \mathrm{ml})$ & $6.8 \pm 1.3$ & $6.9 \pm 1.8$ & $6.6 \pm 1.4$ & $0.717 \pi$ \\
\hline $\mathrm{LH}(\mathrm{mlU} / \mathrm{ml})$ & $5.3 \pm 1.2$ & $5.2 \pm 1.3$ & $5.4 \pm 1.1$ & $0.085 \pi$ \\
\hline E2 (pg/ml) & $63.1 \pm 6.2$ & $61.2 \pm 6.3$ & $60.7 \pm 7.5$ & $0.315 \pi$ \\
\hline Rate of biochemical pregnancy (\%) & $4(8)$ & $5(10)$ & $15(30)$ & $0.001 \S$ \\
\hline
\end{tabular}

Data are presented as mean (SD), ratio or number (\%).

ПOne-way analysis of variance (ANOVA).

$\S$ Pearson chi-squared test.

$\mathrm{P}<0.05$ indicates significant difference between case and control group.

group (4/50) [30\% vs. $10 \% \& 8 \%$ respectively $\mathrm{P}$ - value $=0.001]$.

\section{Discussion}

Endometrial injury is associated with local inflammatory response, cascading a release of pro inflammatory cytokines and growth factors like interleukin-6, leukemia inhibitory factor and tumour necrosis factor $\alpha$. This further induces decidualization and development of endometrium favourable for embryo implantation. Simultaneously, injury modulates gene expression in the endometrium, with upregulation of proimplantation proteins such as mucin 1 transmembrane (MUC1), crystallin alpha $\mathrm{B}$, apolipoprotein D (APOD), phospholipase A2 (PLA2) and Uroplakin Ib (UPIb) [8]. After endometrial injury there is an increase in macrophages and dendritic cells that play an important role in decidualization and implantation. The monocytes recruited to the injured sites are long lived and reside in the tissues for a long time, thereby supporting the effect of injury induced in the preceding cycle to last in the subsequent cycle [9]. Endometrial injury had been studied as a method to improve pregnancy rate in spontaneous cycle [9], IVF (in vitro fertilization) [10] and IUI (intrauterine insemination) [11]. It had been done by different instruments as Pipelle [5] or by using hystroscope [12] and suggested that there is a beneficial effect of endometrial scratching in terms of biochemical pregnancy rate in women with unexplained infertility.

In the present study as shown in (Table 1) and (Figures 3, 4), the rate of biochemical pregnancy, was significantly higher in women subjected to endometrial injury by Pipelle (15/50) compared with non endometrial injury by sound (5/50) and control group (4/50) [30\% vs. $10 \% \& 8 \%$ respectively $\mathrm{P}$ - value $=0.001]$, which agree with two RCTs (including 105 women and 217 women) done by Gibreel study and Parsanezehad study respectively, the biochemical pregnancy rate was significantly higher in women in Pipelle group (16/54) than 


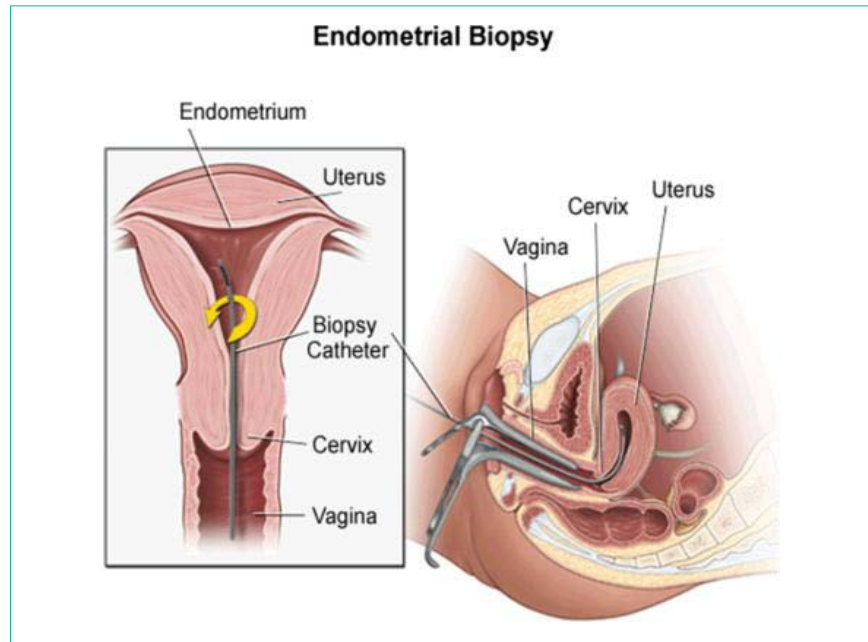

Figure 2: The endometrial biopsy procedure [15].

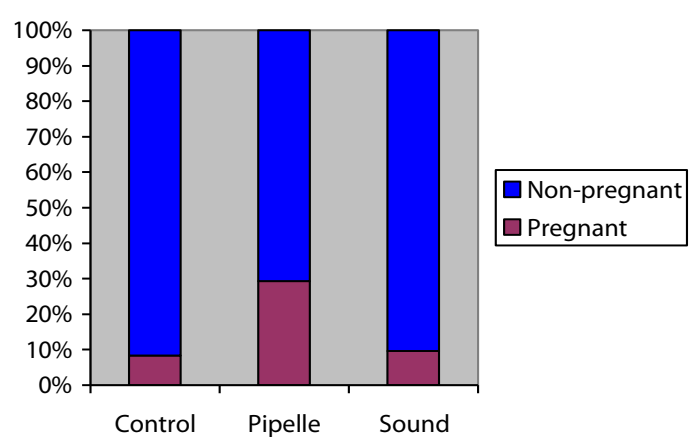

Figure 3: Biochemical pregnancy rate in the control, uterine sound, and pipelle groups.

in women in sound group (6/51) $[29.6 \%$ and $11.7 \%$, respectively, $\mathrm{P}=0.03$ ] [5]. The pregnancy rate was significantly higher in the endometrial injury group compared to the control group [17/114 (14.9\%) vs. 6/103 (5.8\%)] [6]. The pregnancy rate was lower than the pregnancy rate in the present study although Parsanezehad used a large number of populations, this may be due to ovulation induction by gonadotropins that may affect the endometrial morphology and endometrial receptivity because certain integrins expressed by the endometrium seem to be reduced in the glandular epithelium after ovulation induction [13]. But uterine Natural Killer (NK) cells in natural and stimulated cycles in 40 healthy oocyte donors had been studied and demonstrated that the CD56+ NK cell count in the endometrium was reduced by ovarian stimulation but normalized by endometrial injury in the late proliferative phase. These cells present the main source of the endometrial immune regulatory cytokines [14].

Lensen reported that 3 months of expectant management, pregnancy rates of $25 \% v s .9 \%$ in the Pipelle and cotrol respectively [15]. With an adjustment for live birth rates, which are generally $19 \%$ lower than pregnancy rates in a two-sided test, 158 women are required in each arm (316) in total. Mahran mention that Implantation and live birth rates were significantly higher in intervention compared with control group ( $22.4 \%$ vs. $18.7 \%, \mathrm{p}=0.02$ and $67 \%$ vs. $28 \%, \mathrm{p}=0.03)$, respectively on first cycle IVF/ICSI outcome as endometrial injury

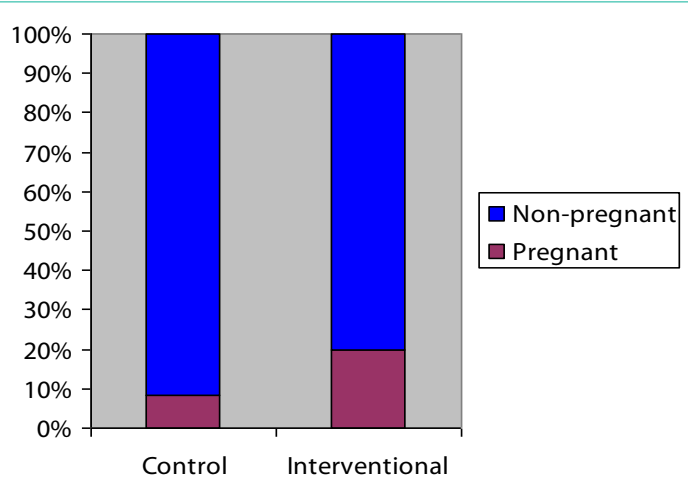

Figure 4: Biochemical pregnancy rate in the control and interventional groups.

was done in the luteal phase of the proceeding cycle preceding the stimulation cycle [16]. Although the underlying mechanism of how endometrial injury improves endometrial receptivity remains unclear, inflammatory background is highly suggested. other randomized, control trial showed that endometrial scratching is useful when it is performed in the mid proliferative phase in increasing biochemical and clinical pregnancy rates after failed previous intra uterine insemination trials $[27 / 106(25.5 \%)$ vs. $15 / 106(14.1 \%) \mathrm{p}=0.03$ and $24 / 106(22.6 \%)$ vs. $12 / 106(11.3 \%) ; \mathrm{p}=0.02]$ [(25.5\%) in endometrial injury group $v s$. control group respectively.

In contrast to our study a recent controlled trial of 93 consecutive subjects done by [17] mention that The chemical, clinical and ongoing pregnancy rates were lower in endometrial injury group $11.8 \%$ compared with control group $20.5 \%$ but no statistically significant. The multiple pregnancy rate and miscarriage rate also were lower in endometrial injury group compared with control group. An explanation was offered by [18] called "backwards development theory". They speculated that Controlled Ovarian Hyperstimulation $(\mathrm{COH})$ negatively affects embryo implantation through histological progression and functional changes such as pinopode maturation advancement and steroid receptor down regulation.

\section{Refernces}

1. Li R, and Hao G. Local injury to the endometrium: Its effect on implantation. Curr Opin Obstet Gynecol. 2009; 21: 236-239.

2. Guven S, Kart C, Unsal M, Yildirim O, Odaci E, Yulug E. Endometrial injury may increase the clinical pregnancy rate in normoresponders underwent long agonist protocol intracytoplasmic sperm injection cycles with single embryo transfer. Fertil Steril. 2011; 96: 277.

3. Karimzadeh M, Oskouian $H$, Ahmadi S, Oskouian L. Local injury to the endometrium on the day of oocyte retrieval has a negative impact on implantation in assisted reproductive cycles: a randomised controlled trial. Arch Gynecol Obstet. 2010; 281: 499-503.

4. Karimzadeh MA, Ayazi Rozbahani M, Tabibnejad N. Endometrial Local injury improves the pregnancy rate among recurrent implantation failure patients undergoing in vitro fertlization/ intra cytoplasmic sperm injection: a randomized clinical trial. Aust N Z J Obstet Gynecol. 2009; 49: 677-680.

5. Gibreel A, Badawy A, El-Refai W, El-Adawi N. Endometrial scratching to improve pregnancy rate in couples with unexplained subfertility: a randomized controlled trial. J Obstet Gynecol Res. 2013; 39: 680-684.

6. Parsanezhad ME, Dadras N, Maharlouei N, Neghahban L, Keramati P, Amini M. Pregnancy rate after endometrial injury in couples with unexplained infertility. Iran J Reprod Med. 2013; 11: 869-874. 
7. Raziel A, Schachter M, Strassburger D, Berno O, Ron-El R, Friedler S Favorable influence of local injury to the endometrium in intracytoplasmic sperm injection patients with high-order implantation failure. Fertil Steril. 2007; 87: 198-201.

8. Kalma Y, Granot I, Gnainsky Y, Czernobilsky B, Dekel N, Barash A, et al. Endometrial biopsy-induced gene modulation: first evidence for the expression of bladder transmembranal uroplakin $\mathrm{lb}$ in human endometrium. Fertil Steril. 2009; 91: 1042-1049

9. Gnainsky Y, Granot I, Aldo P, Barash A, Schechtman E, Mor G, et al. Local injury of the endometrium induces an inflammatory response that promotes successful implantation. Fertil Steril. 2013; 94: 2030-2036.

10. Toukhy T, Sunkara SK, Khalaf S. Local endometrial injury and IVF outcome: a systematic review and meta-analysis. Reproductive BioMedicine. 2012; 25 : 345-354.

11. Abdelhamid AM. The success rate of pregnancy in IUI cycles following endometrial sampling. Arch Gynecol Obstet. 2013; 288: 673-678.

12. Makrakis E, Hassiakos D, Stathis D, Vaxevanoglou T, Orfanoudaki E. Hysteroscopy in women with implantation failures after in vitro fertilization: findings and effect on subsequent pregnancy rates .J. Minim. Invasive Gynecol. 2009; 16: 181-187.

13. Shapiro BS, Daneshmand ST, Garner FC, Aguirre M, Hudson C, Thomas S Evidence of impaired endometrial receptivity after ovarian stimulation for in vitro fertilization: a prospective randomized trial comparing fresh and frozenthawed embryo transfer in normal responders. Fertil Steril. 2011; 96: 344 348
14. Junovich G, Mayer Y, Azpiroz A, Daher S, Iglesias, A, Zylverstein C, et al. Ovarian stimulation affects the levels of regulatory endometrial NK cells and angiogenic cytokine VEGF. Am J Reprod Immunol. 2011; 65: 146-153.

15. Lensen S, Martins W, Nastri C, Farquhar C, Lynn Sadler. Pipelle fo Pregnancy (PIP): study protocols for three randomised controlled trials. Trials. 2016; 17: 216

16. Mahran A, Ibrahim M, Bahaa $\mathrm{H}$. The effect of endometrial injury on first cycle IVF/ICSI outcome: A randomized controlled trial. Int J Reprod BioMed. 2016; 14: 193-198.

17. Abbas Aflatoonian, Ramesh Baradaran Bagheri, Robabe Hosseinisadat The effect of endometrial injury on pregnancy rate in frozen-thawed embryo transfer: A randomized control trial. Int J Reprod BioMed. 2016; 14: 453-458.

18. Zhou L, Li R, Wang R, Huang HX, Zhong K. Local injury to the endometrium in controlled ovarian hyperstimulation cycles improves implantation rates. Fertil Steril. 2008; 89: 1166-1176.

19. Siristatidis C, Vrachnis N, Vogiatzi P, Charalampos C, Retamar AQ, Bettocch $\mathrm{S}$, et al. Potential Pathophysiological Mechanisms of the Beneficial Role of Endometrial Injury in In-Vitro Fertilization Outcome. Reproductive science. 2014; 21: 955-965
Austin J Obstet Gynecol - Volume 4 Issue 5 - 2017

Submit your Manuscript | www.austinpublishinggroup.com

Belal et al. (C) All rights are reserved
Citation: Belal S, Nagy OAEFA, Elwanis MA and Mahmod H. The Effect of Endometrial Scratching by Pipelle on the Pregnancy Rate in Couples with Unexplained Infertility. Austin J Obstet Gynecol. 2017; 4(5): 1087 